\title{
Childhood maltreatment and its effect on neurocognitive functioning: Timing and chronicity matter
}

\author{
RAQUEL A. COWELL,${ }^{a, b}$ DANTE CICCHETTI, ${ }^{b, c}$ FRED A. ROGOSCH ${ }^{c}$ AND SHEREE L. TOTH ${ }^{c}$ \\ ${ }^{a}$ St. Norbert College ${ }^{b}$ University of Minnesota Institute of Child Development; and ${ }^{c}$ University of Rochester Mt. Hope Family Center
}

\begin{abstract}
Childhood maltreatment represents a complex stressor, with the developmental timing, duration, frequency, and type of maltreatment varying with each child (Barnett, Manly, \& Cicchetti, 1993; Cicchetti \& Manly, 2001). Multiple brain regions and neural circuits are disrupted by the experience of child maltreatment (Cicchetti \& Toth, in press; DeBellis et al., 2002; McCrory \& Viding, 2010; Teicher, Anderson, \& Polcari, 2012). These neurobiological compromises indicate the impairment of a number of important cognitive functions, including working memory and inhibitory control. The present study extends prior research by examining the effect of childhood maltreatment on neurocognitive functioning based on developmental timing of maltreatment, including onset, chronicity, and recency, in a sample of 3- to 9-year-old nonmaltreated $(n=136)$ and maltreated children $(n=223)$. Maltreated children performed more poorly on inhibitory control and working-memory tasks than did nonmaltreated children. Group differences between maltreated children based on the timing of maltreatment and the chronicity of maltreatment also were evident. Specifically, children who were maltreated during infancy, and children with a chronic history of maltreatment, exhibited significantly poorer inhibitory control and working-memory performance than did children without a history of maltreatment. The results suggest that maltreatment occurring during infancy, a period of major brain organization, disrupts normative structure and function, and these deficits are further instantiated by the prolonged stress of chronic maltreatment during the early years of life.
\end{abstract}

Throughout the course of development, self-control is associated with a wide array of important outcomes, including relationship stability, academic competence, criminal behaviors, and psychopathology. The significance of self-regulatory abilities is increasingly recognized as important to the overall well-being of both the individual and society. Yet knowledge on best practices for fostering self-control, including a focus on critical time points for nurturing optimal development, must still be identified. It is generally understood that early adverse life experiences, such as the occurrence of child maltreatment, often carry long-lasting consequences (Cicchetti \& Valentino, 2006; Sroufe, 1990; Sroufe, Egeland, Carlson, \& Collins, 2005).

Maltreatment in early childhood has been shown to have cascading effects on neurobiological, social, emotional, and

This research was supported by grants from the National Institute of Drug Abuse (R01DA017741), the National Institute of Mental Health (R01MH083979), and the Spunk Fund, Inc. (to D.C.). Raquel A. Cowell's work was supported by grants from the National Institutes of Health under Ruth L. Kirschstein National Research Service Award from the NICHD and the University of Minnesota Doctoral Dissertation Fellowship (5T32HD007151). We are grateful to the children and families who participated in this research. We appreciate the generous collegiality of Adele Diamond, who graciously provided us with her measures and training materials and reviewed our pilot videotapes to insure that our work was appropriately conducted.

Address correspondence and reprint requests to: Raquel A. Cowell, St. Norbert College, Mail Stop 07-3A, 100 Grant Street, De Pere, WI 54115; E-mail: raquel.cowell@ snc.edu; or Dante Cicchetti, Institute of Child Development, University of Minnesota, 51 East River Road, Minneapolis, MN 55455; E-mail; cicchett@umn.edu. cognitive development. Childhood maltreatment is multidimensional and represents a failure of the child's environment (e.g., familial and societal) to provide for the child's basic needs for safety, security, and support (Cicchetti \& Lynch, 1993). Childhood maltreatment includes neglect, emotional abuse, physical abuse, and/or sexual abuse. Maltreated children commonly experience more than one type of maltreatment, either concurrently or separately (Barnett, Manly, \& Cicchetti, 1993).

Researchers, clinicians, and policymakers would benefit from a clearer understanding of how perturbations to one's early environment may disrupt the ability to self-regulate later on (Cicchetti \& Tucker, 1994). Studies of maltreated children's adaptation efforts informs us about the range and variability of individual response to challenge and adversity and helps to specify the limits of biological and behavioral plasticity. Examinations of maltreated children can provide an entree into the study of the organization, disorganization, and reorganization of multiple developmental systems (Cicchetti \& Toth, 1995). Certain experiences, such as the atypical childrearing characteristic of maltreating parents, may carry greater weight based on their timing, type, and duration relative to other experiences. Focusing on both the nature and the timing of such factors, and examining their subsequent relations to outcomes would advance the discussion of the role of early experience on self-control processes by more clearly specifying how early maltreatment experiences differentially affect this ability.

There has been a burgeoning of interest in comprehending how early adverse experiences, such as child maltreatment, 
exert their effects on the developing brain (Lupien, McEwen, Gunnar, \& Heim, 2009). Growing evidence now exists that specific brain regions are affected by child maltreatment. (Cicchetti \& Toth, in press; DeBellis, 2001, 2005; Hart \& Rubia, 2012; McCrory, De Brito, \& Viding, 2010). Multiple brain regions and neural circuits are disrupted by maltreatment experiences. The aberrant neuronal circuitry most likely contributes to the multifinal phenotypes observed in maltreated individuals (Cicchetti \& Rogosch, 1996).

The pathways most affected in maltreated children and adolescents are predominately in fronto-limbic networks. These include the prefrontal cortex (PFC), both the orbitofrontal cortex and anterior cingulate cortex, and the amygdala (DeBellis, 2001; Hart \& Rubia, 2012; McCrory et al., 2010; Teicher et al., 2012). Diffusion tensor imaging studies have revealed deficits in structural connectivity between the anterior cingulate cortex and the dorsolateral, orbitofrontal, and ventromedial prefrontal cortices (Hart \& Rubia, 2012). The atypical activation in these brain regions occurs during response inhibition, workingmemory, and emotion-processing tasks, suggesting disruption of executive function neural networks. Executive functions refer to cognitive competencies that are important for reasoning, planning, and problem solving (Blair, Raver, \& Berry, 2014). Specific working-memory, inhibitory control, and attentionalcognitive abilities comprise executive functions in the developmental cognitive neuroscience literature (Blair et al., 2014; Zelazo \& Bauer, 2013). Early child neglect has been shown to be related to diffuse organization of white matter microstructure in PFC in adolescents, and this has been associated with neurocognitive deficits on tasks that involve assessments of cognitive control, behavioral regulation, and spatial planning (Hanson et al., 2013).

The examination of self-control from different perspectives and time points in developmental research has led to a growing realization that large societal problems such as criminality often begin with individual difficulties in self-control (Moffitt et al., 2011). Willfully controlling one's behavior to accomplish a desired goal is a fundamental skill necessary for long-term success across multiple domains, including meaningful social interactions and cognitive competencies. In a longitudinal study conducted with a cohort of 1,000 children, low self-control was associated with poorer physical health, increased substance dependence, greater financial struggles, and lower income in adulthood (Moffitt et al., 2011). Children with lower self-control also are more vulnerable to negative developmental outcomes in adolescence, such as a greater likelihood of becoming a teenage parent or dropping out of high school (Moffitt et al., 2011). Self-control is considered a relatively stable trait across the lifespan (Casey et al., 2011), and understanding whether and how self-control can be influenced during development represents a significant area for researchers, clinicians, and policymakers alike.

Resisting temptation, achieving long-term goals, or having meaningful relationships depends on an individual's ability to actively control his or her actions to achieve a desired outcome. Broadly defined, self-control is closely related to executive function and cognitive control, and corresponds to the vertical neurocognitive processes of goal-directed control of thought, action, and emotion (Zelazo et al., 2013). Situations requiring control may vary from childhood to adulthood. Childhood examples may consist of not touching anything in the store, listening quietly to the teacher, and sharing a toy with a friend, whereas adulthood examples may include completing a challenging class or stopping long enough to hear a close friend's side of the story. All of these childhood and adulthood examples require a basic level of self-control. Self-control may be expressed differently across the life span; however, continuity in similar self-control tasks across different developmental periods has been empirically supported (Berman et al., 2013; Casey et al., 2011). Childhood performance has been shown to be correlated with adult performance, up to 40 years later (Casey et al., 2011; Eigsti et al., 2006; Mischel et al., 2010). Such continuity in self-control indicates the continued importance of self-control to academic success, personal relationships, citizenship, and mental health.

Neural areas of interest in research on self-control include the right inferior frontal cortex, the superior frontal gyrus, the anterior cingulate cortex, the caudate, and the ventral striatum (Berman et al., 2013; Casey et al., 2011; Hare et al., 2008). Across different stages of development and different selfcontrol tasks, the aforementioned regions show differences in activity based on level of self-control performance. Likely, any compromises to these areas due to early perturbations in the environment carry behavioral consequences in the form of less efficient self-control performance. The extent to which the maturation and efficiency of these structures are susceptible to experiencing negative environmental stressors will provide insight into whether and how early adversity disrupts neural systems and influences individual differences in how experiences are processed. The utilization of a neuropsychological perspective will provide a window into the mechanisms underlying why self-control shows a relatively stable level of continuity across development.

Developmental neuroscientists generally agree that the brain is more malleable at early stages of development, with the greatest amount of growth and change happening prenatally and during the first year of life (Fox, Levitt, \& Nelson, 2010; Stiles \& Jernigan, 2010; Thompson \& Nelson, 2001). It also is thought that different behaviors rely on varied distributions of neural networks and that many of the neural structures within these networks are more vulnerable to disruptions in a child's early environment than during other developmental periods. In animal studies, early life stress has been found to be associated with compromised function of the PFC in rodents (Sullivan, 2003) and neurochemical alterations in Bonnet macaques (Mathew et al., 2002). These studies indicate that early deprivation carries with it longterm consequences in areas of the brain important for self-control. The vulnerability to stress of the PFC in rodents appears to be similarly compromised by stressful experiences in humans (McDermott, Westerlund, Zeanah, Nelson, \& Fox, 2012). 
Specifically, in a sample of children with a history of early adversity (i.e., institutionalized children), an inhibitory control task was performed by never-institutionalized or foster care children and institutionalized children. The institutionalized children had lower inhibitory control scores (a key component of self-control). Those with a history of being institutionalized also displayed the smallest difference in reactivity between correct and error trials via the error-related negativity brain wave difference score, indicating that they processed mistakes more slowly or less accurately than their more fortunate counterparts (McDermott et al., 2012). This delay in processing time highlights the importance of processing speed in relation to self-control and elucidates the importance of approaching the study of self-control from multiple perspectives. In addition, in children internationally adopted from institutions, current research has shown patterns of reduced glucose metabolism in regions of the frontal cortex, including the orbitofrontal gyrus and infralimbic PFC, compared to noninstitutionalized youth (Chugani et al, 2001). Children with an early history of institutionalization also exhibit lower levels of electrical brain activity than their same-aged counterparts (Marshall \& Fox, 2004). This work emphasizes the importance of considering early life stressors as disruptive agents that expose some affected individuals to the associated problems of low self-control.

Self-control can be conceptualized as a general skill, with the nature of its acquisition and implementation being represented differently across development. The investigation of disruptions to its development, such as those experienced by maltreated children, could inform the points in time during which processes relevant to self-control are emerging (Johnson, 2011). Self-control is a concept encompassing top-down neurocognitive processes involved in explicit, goal-directed processes (Weintraub et al., 2013). In parallel with the PFC, self-control develops rapidly during the preschool years (Zelazo, Carlson, \& Kesek, 2008). Inhibitory control, cognitive flexibility, and working memory are all processes involved in self-control (Weintraub et al., 2013). Although the nature of the self-control skills being acquired at a certain point in development may be more or less sophisticated, the challenge to the brain may be similar (Johnson, 2011). It is possible that vertical interactions (i.e., top-down processes), more than horizontal interactions, are negatively impacted by the experience of maltreatment. The current research will explore whether basic self-control processes are similarly impacted by maltreatment or whether the negative effects of maltreatment are confined to prefrontally mediated areas, which are considered important for top-down processes such as rule following and goal-directed behavior (Johnson, 2011).

Observing similarities and differences in working memory, inhibitory control, memory, and motor control between maltreated and nonmaltreated children is only useful if the differences in these areas are categorized in a meaningful way. Because childhood maltreatment is a multicomponent construct, encompassing instances of physical, sexual, and emotional abuse as well physical and emotional neglect, clearly defining subtypes of maltreatment experienced is critical in order to make meaningful comparisons between maltreated and nonmaltreated children. For example, by examining potential differences in maltreatment chronicity, and gaining a clearer perspective of whether the number of developmental periods in which maltreatment occurs matters, it is possible to gain a better understanding of the resilience of a system, as well as how recurring traumatic experiences impact maltreated children at different points in time (Cicchetti, 2013).

Children with a history of foster care are another group who have experienced early adversity, predominantly through neglect, abuse, caregiver disruptions, or a combination of these situations. A recent functional magnetic resonance imaging study of maltreated children in foster care showed atypical neuronal activation during an inhibitory control task (Bruce et al., 2013). Foster care children showed more diffuse patterns of activation, and displayed stronger activation in the left inferior parietal lobule and the right superior occipital cortex; moreover, compared to nonmaltreated children, they also demonstrated less activation in regions typically associated with inhibitory control performance, such as the anterior cingulate cortex (Bruce et al., 2013). One general theory that may lend insight into why atypical activation occurs in these regions is Johnson's (2011) proposed model of interactive specialization, which states that some cortical regions have broad functionality and their functions do not become well defined until, through a sufficient amount of activation and successful competition with other regions, they become functionally efficient. This process is activity dependent and requires a satisfactory amount of input to develop appropriately. Following from an interpretation of interactive specialization, this suggests that regions typically involved in self-control may not be receiving important activity-dependent interactions necessary for neuronal maturation and specialization (Johnson, 2011). Alternatively, these systems may be exposed to a wide array of activities but in a disruptive and unpredictable manner.

Although the relationship among experience, neuronal development, and behavior has yet to be clearly understood, discovering ways that maltreatment interferes with biological and behavioral developmental processes can further clarify when the system is most vulnerable.

In the current study, we sought to provide insight into how the differential effects of the timing, chronicity, and subtypes of maltreatment impact different components of self-control by examining the cognitive components considered important for self-control, including working memory and inhibitory control. Through examining the performance on several tasks related to self-control in children with and without a history of maltreatment, it is expected that environmental influences on self-control in the earlier stages of development will be elucidated. Because maltreatment is a pervasive and prevalent occurrence in the United States (Sedlak et al., 2010), a clearer understanding of how it impacts self-control likely will have far-reaching implications.

The objective of the present study was to investigate potential performance differences between maltreated and 
nonmaltreated children on a battery of neurocognitive tasks. It was expected that maltreated children would show the greatest detriment in performance on tasks involving inhibitory control and working memory, tasks thought to rely on prefrontally mediated regions (Diamond, Prevor, Callender, \& Druin, 1997). Differences based on the chronicity of maltreatment also were predicted, with children experiencing chronic maltreatment expected to show greater neurocognitive performance decrements compared to nonmaltreated children or children who experienced maltreatment for a more abbreviated period of time. The effects of maltreatment subtype also were examined as predictors of neurocognitive performance.

\section{Method}

\section{Participants}

The sample included 228 maltreated low socioeconomic status (SES) children (89 females, 139 males) and 142 nonmaltreated low-SES comparison children (60 females, 82 males) across six age groupings: 3 years ( $M=3$ years, 7 months), 4 years $(M=4$ years, 7 months), 5 years $(M=5$ years, 5 months), 6 years ( $M=6$ years, 6 months $), 7$ years $(M=7$ years, 5 months), and 8 to 9 years ( $M=8$ years, 5 months). The maltreated and nonmaltreated children were from an urban upstate New York area. Children with a history of maltreatment were selected from the population of children determined by the local Department of Human Services (DHS) to have experienced child abuse and/or neglect. The nonmaltreated children were demographically similar and recruited from the same neighborhoods as the maltreated children.

The demographic features were comparable between the nonmaltreated and maltreated children, including gender, $\chi^{2}$ $(1,370),=0.38$, $n s$ (57.7\% vs. $61.0 \%$ male), and minority race/ethnicity $\chi^{2}(1,370)=0.77, n s(73.0 \%$ vs. $77.1 \%)$, for nonmaltreated and maltreated groups, respectively. The nonmaltreated and maltreated groups also were comparable in terms of family income (in thousands), $t(354)=0.78, n s$ ( $M$ $=19.01, S D=10.9$ vs. $M=18.11, S D=10.3)$, represented in the two lowest levels of the Hollingshead scale of SES, $\chi^{2}$ $(1,360)=1.47$, ns $(74.5 \%$ vs. $79.9 \%)$, and history of receipt of public assistance, $\chi^{2}(1,358)=0.84, n s(91.5 \%$ vs. $94.0 \%)$.

All parents provided informed consent for their child's participation and for examination of any DHS records regarding the family. Those families identified as having a child between the ages of 3 and 9 with a documented history of child maltreatment in the family were contacted by a DHS liaison. Permission was first obtained from consenting families for project staff to contact them about participating in the study. Maltreatment status was determined through a detailed examination of Child Protective Service (CPS) and Preventive Services records at DHS. Children recruited for the maltreatment group had been identified by DHS as having experienced child abuse or neglect. The maltreated sample was representative of families receiving services at the country DHS with respect to lowincome and minority racial/ethnic status. In addition to DHS records, mothers were interviewed with the Maternal Child Maltreatment Classification Interview (Cicchetti, Toth, \& Manly, 2003) to determine other maltreatment experiences that may not have been contained in DHS records.

The nonmaltreated, low-SES comparison group were recruited from families receiving Temporary Assistance to Needy Families. Because maltreatment occurs predominantly in the low-SES population, families receiving Temporary Assistance to Needy Families were selected based on their similarity to the demographic characteristics of the families with maltreated children. Searches to assure the absence of CPS or Preventive Services records were conducted to confirm nonmaltreatment status. Nonmaltreatment status was further verified by maternal report, using the MMCI interview. Families were excluded from the nonmaltreatment group if there was any family history of DHS involvement as indicated by record searches or maternal report.

All existing DHS records for these families were screened and coded by raters using the Barnett et al. (2003) nosological classification system for child maltreatment. An assessment of maltreatment history could include reports made by caregivers, teachers, CPS workers, and other community members (e.g., neighbors, physicians, police, and daycare providers). This system has been previously reported as reliable and valid in classifying incidents of maltreatment and in differentiating subtypes of maltreatment (e.g., Bolger \& Patterson, 2001; Manly, Kim, Rogosch, \& Cichetti, 2001). Trained research staff coded the DHS records, and adequate reliability was obtained (average intraclass correlations ranged from 0.82 to 1.0 ).

The maltreatment subtypes, based on the Barnett et al. (1993) classification system, included sexual abuse, coded when any sexual contact or attempted sexual conduct occurred between a child and an adult. Sexual abuse incidents ranged from exposure to inappropriate sexual activities to forced intercourse. Physical abuse was coded if DHS records indicated that a caregiver had inflicted a physical injury on a child by nonaccidental means. Coded incidents ranged from corporal punishment that was deemed excessive because of bruising to permanently disfiguring injuries, such as broken bones. Neglect was coded if DHS records indicated that a caregiver had failed to provide minimal care in meeting the child's physical needs or the necessary supervision to ensure the child's safety in and out of the home. Examples of coded neglect included leaving a young child alone, failing to maintain sanitary living conditions, and lack of providing adequate nourishment. Emotional maltreatment was coded for occurrences involving persistent or extreme thwarting of children's emotional needs. Examples of coded emotional maltreatment included serious threats to injure a child, caregivers' attempts to commit suicide in the presence of the child, and repeated berating of the child.

The experiences of abuse and neglect with the maltreated sample were heterogeneous. In this maltreated sample, $66 \%$ had been neglected, $57 \%$ had experienced emotional maltreatment, $24 \%$ had been physically abused, and $7 \%$ of had been sexually abused. Within the maltreated sample, $11 \%$ 
of children did not have sufficient detail in the family DHS records to code subtype. Over half (54\%) of maltreated children had experienced multiple subtypes of maltreatment. This finding is consistent with other samples of maltreated children as well as the nature of child maltreatment (Barnett et al., 1993; Cicchetti \& Barnett, 1991).

\section{Procedures}

All children were tested on 10 tasks ( 3 working-memory and inhibition tasks, 4 memory tasks, and 2 motor tasks). For all cognitive neuropsychological testing, both a tester and an assistant were present. Sessions were videotaped for additional coding. The testing session lasted approximately $1.5 \mathrm{hr}$. The procedures for the 10 neuropsychological tests used with the 3- to 9-year-olds are described below.

Children were administered the day-night Stroop-like task and the control Stroop-like task (in random order) first, followed by the six boxes task in which the boxes remain stationary, the six boxes task in which the boxes are scrambled after each reach, the Corsi-Milner test of short-term temporal memory, the Corsi-Milner test of short-term recognition memory, the tapping test, global-local spatial processing (forced choice reaction time procedure), the three pegs task, and the line bisection task.

Stimulus presentation times, delay durations, fixation times, and response latencies were verified or determined from the videotape records of each session. Online coding of the participant's and experimenter's actions, such as response accuracy, was always rechecked by subsequent coding of the videotape to confirm accurate coding. Any discrepancies were resolved to consensus. The administrators and videotape coders were unaware of group membership of the children.

\section{Measures}

\section{Inhibitory control and working-memory tasks.}

The day-night Stroop-like task. The day-night Stroop-like task (Gerstadt, Hong, \& Diamond, 1994) requires participants to hold two rules in mind. To successfully complete this task, the child had to inhibit what the stimulus represented and say the opposite. For example, when the child viewed a picture of the sun, he or she was to say "night," and when he or she saw a picture of the moon and stars, he or she was to say "day."

The day cards were made up of nine black cards with a yellow moon and gold stars, and the night cards were made up of nine white cards with a yellow sun. Each session consisted of a pseudorandom series of 16 trials, with no more than three of a kind in a row. The dependent variables were response accuracy and latency. Response latency was measured from the time the child first saw the day or night card until he or she gave a verbal response.

Before testing, the child had to first demonstrate an understanding of the directions by recognizing at least 1 day and 1 night card in a row. The child needed to have answered each rule correctly at least once during either of the two practice trials or the first two trials of testing for his or her data to be considered usable.

The tapping task. The tapping task (Diamond \& Taylor, 1996) required the child to engage simultaneously in two processes. He or she had to hold two rules in mind at once and also inhibit the tendency to mimic the experimenter's actions. A pseudorandom series of 16 trials with no more than three of a kind in a row were presented. To successfully complete the task, the child was instructed to tap once with his or her wooden dowel whenever the experimenter tapped twice with his or her wooden dowel, and to tap twice with his or her wooden dowel whenever the experimenter tapped once with his or her wooden dowel. Immediately after receiving each instruction, the child was allowed to practice each rule. A brief pretest was administered. Trial by trial performance was recorded for both children who passed and children who failed the pretest. The dependent variable was the number of correct taps. For each trial, the experimenter tapped first and then handed the dowel to the child to tap. One dowel was shared between the experimenter and the child to prevent either party from tapping before the other party finished.

The three pegs task. Similar to the tapping task, the three pegs task (Balamore \& Wozniak, 1984) required the child to inhibit a prepotent response. In this task, the child was shown a pegboard containing three pegs arranged in the order red, yellow, green. The child was instructed to tap the pegs in the order red, green, yellow. The successful completion of this task required the child to inhibit the tendency to tap the pegs in their spatial order and follow the instruction to tap the pegs according to their color. There were three possible conditions present in this task, and the dependent variable was the number of conditions the child needed to successfully complete the pegs task.

The first condition was the verbal instruction condition. In this condition, the experimenter said slowly and clearly, "With the hammer, I want you to tap each peg one time, the red peg first, then the green peg, and then the yellow peg." After speaking, the instructor gave the hammer to the child. If the child tapped the pegs in the correct order, he or she was praised and asked to do it again. If the child successfully completed the confirmation trial, then testing ended.

If the child was wrong on either of these two trials, then the second condition demonstration plus verbal instruction was administered. In this condition, the experimenter instructed the child to watch him or her, and while tapping the appropriate peg, the experimenter provided the same verbal instructions as before. After this demonstration, he or she handed the hammer back to the child. If the child tapped the pegs in the correct order, he or she was praised and asked to do it again. If the child successfully completed the confirmation trial, then testing was concluded. 
Like before, if the child was wrong on either of these two trials, then the third verbal condition was administered. The experimenter administered the instructions similar to the second condition and provided additional guidance by saying, "With the hammer, tap the red peg first, then the green peg, and then the yellow peg. But this time, I want you to say the colors at the same time you tap the pegs, like this ..." After this demonstration, the experimenter handed the hammer back to the child. If the child tapped the pegs in the wrong order, then testing ended. If the child was correct, then a confirmation trial was administered.

\section{Memory tasks.}

Corsi-Milner test of temporal order and recognition memory. In the Corsi-Milner test of temporal order and recognition memory, children were shown a series of pictures sequentially, with each picture on its own card. At certain intervals, participants were shown two cards, and they were asked one of two questions: for temporal order memory, children were asked to identify which of the two pictures they had last seen, and for recognition memory, children were asked to identify which of the two pictures they already saw. The temporal order question required the children to keep track of the order in which the stimuli were presented. The recognition question required the children to identify which of the two pictures, regardless of order, they had already seen.

Prior to testing, the child had to demonstrate an understanding of the words seen last. To do this, the experimenter showed the child one picture card, then another, and then two cards. The child was asked, "Which of these two pictures did you see last?" Children needed to be correct on two of these practice trials for their data to be considered valid. The dependent variable was the number of questions answered correctly.

After the practice trials, children were shown 1 card at a time for $1 \mathrm{~s}$ each. After the first 10 cards, the child was presented with 2 cards and asked to identify which of the two pictures he or she last saw. Then 5 more cards were shown to the child. This was followed with 2 cards, and the child was asked to identify which of the two pictures he or she had already seen. These two questions were alternated after every five single-object presentations until Trial 30. Then these two questions were alternated after every 2 pictures until 40 pictures total had been shown to the child and five of each type of question was asked.

The six boxes task (boxes scrambled after each reach). The six boxes task (boxes scrambled after each reach) is a working-memory task that requires participants to keep track of which boxes they have already opened. The six boxes used were identical in shape and size, but each was topped with a different sticker. Before the start of the task, the child was allowed to pick out six stickers. He or she was then instructed to place one sticker into each of the six boxes. Once the boxes were baited, the child was allowed to reach into any box in any order, but could only open one at a time. Between reaches, a delay was imposed, and the six boxes were scrambled behind a screen that blocked the child's view. After each scramble, the child was again allowed to reach for any of the six boxes. He or she was penalized if he or she reached for a box that had already been chosen (i.e., there was no sticker inside). The dependent variable used was the number of reaches completed by the child before all six boxes were opened.

The six boxes task (boxes remain stationary). The six boxes task (boxes remain stationary) requires participants to keep track of which boxes they have already chosen. While it is similar to the six boxes task described above, in this version the boxes remain in the same spatial location for the duration of the task. The dependent variable was the number of reaches completed by the child before all six boxes were opened.

\section{Attention and motor control tasks.}

The control version of the day-night Stroop-like task. The control version of the day-night Stroop-like task (Gerstadt et al., 1994) also required participants to hold two rules in mind; however, an inhibitory response was not expected in the Stroop-like task because abstract designs were used. Children were instructed to say "day" to the card with the squiggle design and "night" to the card with the checkerboard design. Except for the design on the cards, all procedures used in the control task were identical to the procedures used in the Stroop-like task.

The global-local spatial processing task (forced choice procedure). The global-local spatial processing task (forced choice procedure) requires attention to both smaller items that form a larger stimulus and to the outline of the larger stimulus. Children are first shown the composite stimulus (e.g., an $\mathrm{H}$ made up of S's) for $2 \mathrm{~s}$. Then they are shown the global or local component of that stimulus paired with something else (e.g., an $\mathrm{H}$ and a V) and are asked to point, as fast as they can, to the choice that looks like that first picture or that looks like things that were in that first picture. Then the children are shown the other component of the composite stimulus paired with something else (e.g., an S and a G) and are again asked to point. There were 12 trials, and response latency was the dependent measure.

The line bisection task. The line bisection task measures spatial perception. Participants were given 12 sheets of paper with lines drawn on them and asked to find the midpoints of the lines. The pages were presented one at a time, one line per page. The lines varied in length and position on the page. The child was asked to put a mark at the middle of each line as the sheets were presented. The experimenter demonstrated the procedure once before testing, and no feedback was given. The dependent variable was the amount the child deviated from the midpoint of each line. 


\section{Results}

\section{Pretest performance}

Initially, chi-squared analyses were conducted to assess performance differences on tasks that include pretests in order to ascertain whether children conceptually understood the task. Pretests were involved from the following subtests: the tapping task, the three pegs task, the day-night Stroop-like task, and the control Stroop task. At age 4, maltreated children (76.3\%) failed to pass the tapping task significantly more often than the nonmaltreated comparison children $(40.9 \%), \chi^{2}(1$, $65)=8.174, p=.004$; see Figure 1 for group failure rates on the tapping task). With regard to performance differences based on age, 3-year-olds from both groups had a greater rate of failure than any other age group on the tapping task, the three pegs task, the day-night Stroop-like task, and the control Stroop task. Individuals who did not pass one of the pretests were retained in the overall analyses, while their performance on tasks for which they failed the pretest was excluded.

Performance based on a child's age in years at time of test was examined and found to be significant, $F(5,358)=$ 29.92, $p=.00, \eta^{2}=0.30$. To allow for an examination of cognitive performance over and above the effects of age, a participant's age in months was included as a continuous covariate for all following analyses.

\section{Cognitive function task performance}

Three components of cognitive function, including inhibitory control/working memory (ICWM), memory, motor control/ attention, and a cognitive function composite (CFC) were used to examine between-group differences. These areas were determined by the underlying neural structures implicated in these tasks (see Diamond et al., 1997).

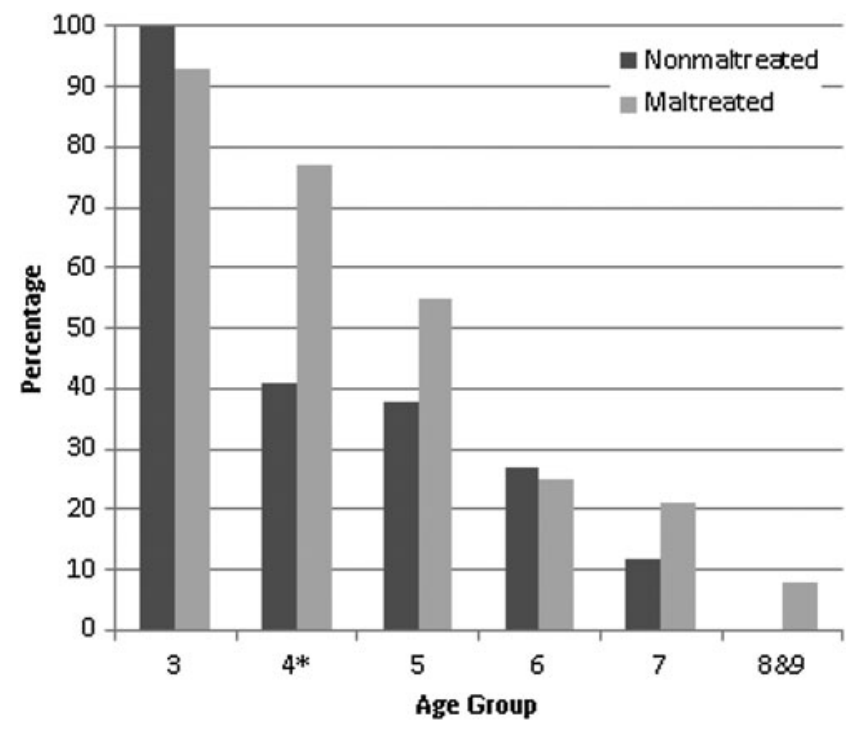

Figure 1. Pretest failure rates on tapping task for nonmaltreated and maltreated children.
The $Z$ scores were computed for performance on task-relevant summary variables, and these standardized variables were then used to generate composites for the ICWM, memory, motor control/attention, and the CFC. (See Table 1 for the number of tasks included in each composite score.) The relevant summary variables for the ICWM composite consisted of the number of correct taps in the tapping task, the number of levels correctly passed in the three pegs task, and the number of correct trials in the day-night Stroop-like task. To be included, children had to pass/complete two of the three ICWM tasks (a $67 \%$ pass rate). Included children had an ICWM score consisting of his or her standardized performance on each of the relevant ICWM variables assigned. By allowing children who passed at least two of the three ICWM tasks into the analyses, a clearer picture of the construct was provided. For example, if a child had difficulty with only one of the ICWM, then it may be an indication of his or her understanding of that particular task, and not necessarily his or her overall ICWM ability. A one-way between factors analysis of covariance (ANCOVA) with age as a covariate revealed a significant group difference between the maltreated children and the nonmaltreated comparison children, $F(1,358)=9.602, M S E=0.325, p=$ $\left..002, \eta^{2}=.026\right)$. The maltreated children had a significantly lower ICWM score $(M=0.11, S D=0.85)$ than the nonmaltreated comparison children $(M=0.08, S D=0.82)$. The results from the ICWM follow-up tests will be discussed below.

The memory composite included the following relevant summary variables: the number of correct reaches in the Corsi-Milner test (temporal), the number of correct reaches in the Corsi-Milner test (recognition), the number of correct trials in the control Stroop-like task, the number of correct reaches in the six boxes task (scrambled), and the number of correct reaches in the six boxes task (stationary). To be considered, children had to pass/complete three of the five tasks (a $60 \%$ pass rate). Including children with a $60 \%$ pass rate allowed for the examination of general memory performance for children who had difficulty with or did not complete one or two tasks. A one-way between factors ANCOVA with age as a covariate revealed no significant differences between the maltreated children $(M=0.01, S D=0.45)$ and the nonmaltreated comparison children $(M=-0.01, S D=0.39)$ on the memory composite, $F(1,357)=0.192, p=.662, \eta^{2}=0.001$.

The relevant task variables for the motor control/attention composite included the mean response time for global stimuli (reverse scored), and the mean response time for the local stimuli (reverse scored) from the global-local processing task, as well as the mean deviation from the line for the line bisection task. Children had to pass/complete $67 \%$ of the motor control/attention tasks to be included in the analyses. This allowed for the assumption that children had a general understanding of the tasks. A one-way between-factors ANCOVA with age as a covariate revealed no significant differences between the maltreatment children $(M=-0.00, S D=0.58)$ and the nonmaltreated comparison children $(M=-0.00, S D=$ $0.61)$ on the motor control/attention composite, $F(1,369)$ $=0.00, p=.998, \eta^{2}=0.00$. 
Table 1. Types of composite scores and number of component task variables included in each

\begin{tabular}{lccc}
\hline \hline \multicolumn{1}{c}{ Composite Type } & $\begin{array}{c}\text { No. of Component } \\
\text { Task Variables }\end{array}$ & $\begin{array}{c}\text { No. of } \\
\text { Completed Tasks }\end{array}$ & $\begin{array}{c}\text { N Participants } \\
\text { (Maltreated, Comparison) }\end{array}$ \\
\hline $\begin{array}{l}\text { Inhibitory control/working memory } \\
\text { Memory }\end{array}$ & 3 & $2(67 \%)$ & $359(223,136)$ \\
$\begin{array}{l}\text { Attention \& motor control } \\
\begin{array}{l}\text { Cognitive function } \\
\quad \text { composite }\end{array}\end{array}$ & 5 & $3(60 \%)$ & $358(223,135)$ \\
\hline
\end{tabular}

The CFC consisted of chosen variables from all administered tasks. The relevant tasks from each of the previously described subcomponents were included, resulting in 11 variables: the number of correct taps in the tapping task, the number of levels correctly passed in the three pegs task, the number of correct trials in the day-night Stroop-like task, the number of correct reaches in the Corsi-Milner test (temporal), the number of correct reaches in the Corsi-Milner test (recognition), the number of correct trials in the control Stroop-like task, the number of correct reaches in the six boxes task (scrambled), the number of correct reaches in the six boxes task (stationary), the mean response time for global stimuli (reverse scored), the mean response time for the local stimuli (reverse scored) from the global-local processing task, and the mean deviation from the line for the line bisection task. Children had to pass/complete over half or $55 \%$ of the tasks to be included in the CFC. If children could complete half of the CFC, then even if they had difficulty with a particular subcomponent of the CFC, they could still be considered in the analyses. A one-way ANCOVA with age as a covariate revealed no significant differences between the maltreated children $(M=-0.02, S D=0.36)$ and nonmaltreated comparison children $(M=0.02, S D=0.35)$ on the executive function composite, $F(1,358)=1.427, p=.233, \eta^{2}$ $=0.004$. Pass rates differed between task composites to ensure the child was performing above chance for each construct that the specific composite was measuring; because each set of measures varied in the amount of tasks included, the percentages reflected that and also varied slightly.

\section{Follow-up tests}

Follow-up tests examining dimensions of maltreatment were conducted on the ICWM, but not on the other three composites due to lack of significant findings (see Table 2 for the number of participants in each maltreatment group).

A child's performance on the ICWM was examined with regard to the number of maltreatment subtypes the child experienced in his or her lifetime. Children who experienced one or two subtypes of maltreatment were placed into one group ( $n=153$ ), and children who experienced three or four subtypes of maltreatment were placed into a second group $(n=34)$. Both maltreatment groups were compared to the nonmaltreated comparison group $(n=136)$. A one- way ANCOVA with age as a covariate revealed a significant effect for the number of maltreatment subtypes, $F(1,322)=$ $5.890, M S E=0.330, p=.003$. Post hoc follow-up least significant difference (LSD) tests showed that the nonmaltreated comparison children $(M=0.08, S D=0.82)$ performed significantly better than maltreated children experiencing one or two types of maltreatment $(M=-0.12, S D=0.86, p=$ .021 , and those experiencing three or four types of maltreatment $(M=-0.26, S D=0.89, p=.002)$. Maltreated children experiencing one or two types of maltreatment were not significantly different in their performance compared to children experiencing three or four types of maltreatment $(p=.08)$.

The number of developmental periods in which the child was maltreated was used as an index of chronicity. Three separate maltreatment groups were generated, including those who experienced maltreatment during one developmental period $(n=68)$, two developmental periods $(n=47)$, or three or more developmental periods $(n=72)$. All three of these

Table 2. Number of participants included in the ICWM composite for different maltreatment parameter groups

\begin{tabular}{lrr}
\hline \hline Maltreatment Parameter & $n$ & $\%$ \\
\hline Maltreatment status & & \\
$\quad$ Comparison & 136 & 42 \\
$\quad$ Maltreated & 187 & 58 \\
Number of subtypes & & \\
$\quad$ Comparison (0) & 136 & 42 \\
One-two & 153 & 47 \\
Three or more & 34 & 11 \\
Developmental periods & & \\
Chronicity & & \\
Comparison (0) & 136 & 42 \\
One & 68 & 21 \\
Two & 47 & 15 \\
$\quad$ Three or more & 72 & 22 \\
Onset group & & \\
Comparison & 136 & 42 \\
Infancy & 101 & 31 \\
$\quad$ Postinfancy & 86 & 27 \\
Recency group & & \\
Comparison & 136 & 42 \\
Current & 102 & 32 \\
Past & 85 & 26 \\
\hline \hline
\end{tabular}




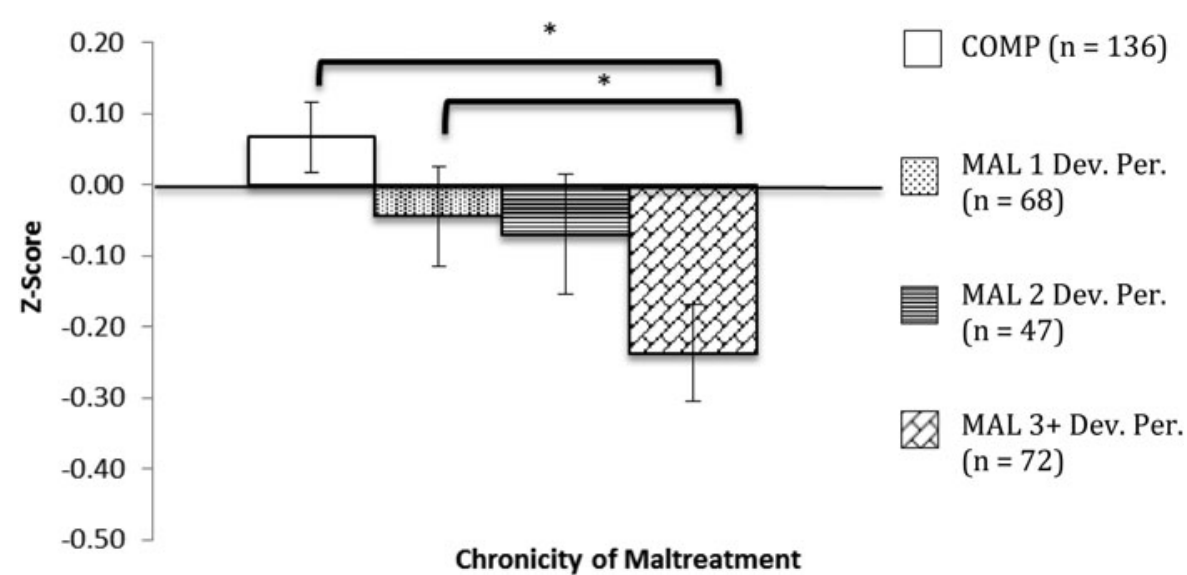

Figure 2. Illustration of performance differences on the inhibitory control/working memory composite based on maltreatment chronicity. Chronically maltreated children performed significantly worse than both their nonmaltreated peers $(p=.00)$ and those experiencing maltreatment during one developmental period $(p=.05)$.

groups were compared to the nonmaltreated comparison children. A one-way ANCOVA with age as a covariate revealed a significant effect for the number of developmental periods in which maltreatment occurred, $F(3,322)=4.385, M S E=$ $0.330, p=.005$. Post hoc follow-up LSD tests revealed that the nonmaltreated comparison group performed significantly better than those children who experienced maltreatment during three or more developmental periods $(\mathrm{M}=-0.42, S D=$ $0.96, p=.000)$, but not children who experienced maltreatment during one $(M=0.07, S D=0.76, n s)$ or two developmental periods $(M=0.02, S D=0.74, n s)$. In addition, those who experienced maltreatment during one developmental period $(M=0.07, S D=0.76)$ performed significantly better than those who experienced maltreatment during three or more developmental periods ( $p=.05$ ), but not those who experienced maltreatment during two developmental periods ( $n s$; see Figure 2 for chronicity of maltreatment means).

Onset of maltreatment was examined in relation to the ICWM. Children whose maltreatment onset occurred during infancy $(n=101)$ were differentiated from those children whose maltreatment onset occurred after infancy $(n=86)$. Both of these groups were compared to the nonmaltreated comparison children. A one-way ANCOVA with age as a covariate revealed a significant effect for the onset of maltreatment, $F(2,322)=6.627, M S E=0.328, p=.002$. Post hoc follow-up LSD tests demonstrated that the nonmaltreated comparison children performed significantly better than those who experienced the onset of maltreatment during infancy $(M=-0.32, S D=0.91, p=.000)$, but not those who experienced the onset after infancy $(M=0.09, S D=$ $0.74, n s)$. In addition, those children who experienced the onset of maltreatment after infancy also performed significantly better than those who experienced the onset of maltreatment during infancy ( $p=.036$; see Figure 3 for onset of maltreatment means).

The recency of a children's maltreatment experience was also considered. Children who had experienced maltreatment in their current developmental period ( $n=103$ ) were differentiated from children who had last experienced maltreatment in an earlier developmental period $(n=85)$. Both of these

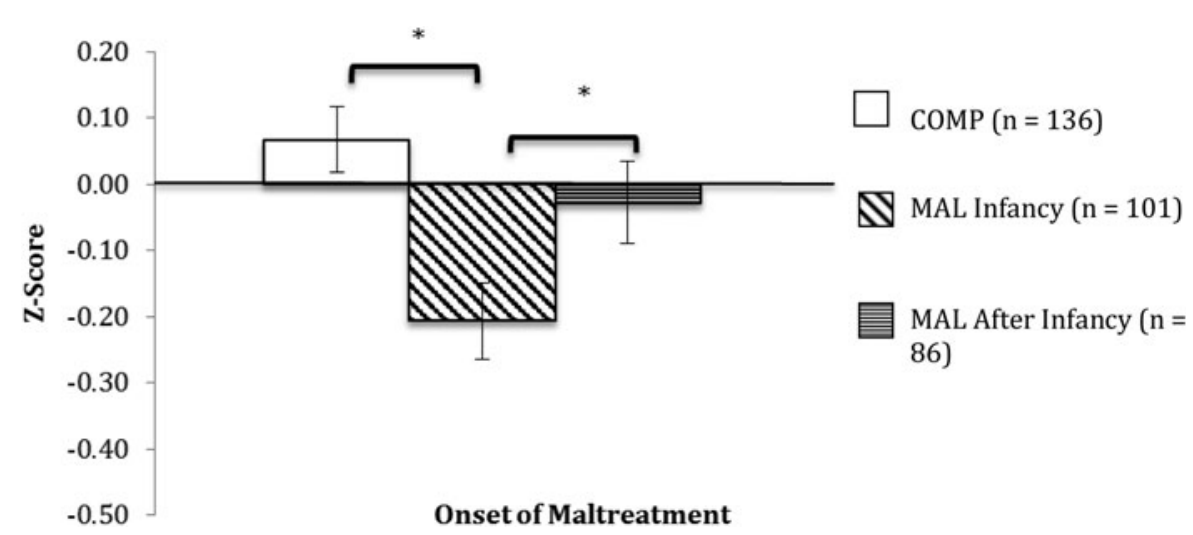

Figure 3. Illustration of performance differences on the inhibitory control/working memory composite based on the onset of maltreatment. Children who first experienced maltreatment during infancy performed significantly worse than both nonmaltreated children and children who did not experience maltreatment until after infancy. 
groups were compared to the nonmaltreated comparison children. A one-way ANCOVA with age as a covariate revealed a significant effect for the recency of children's maltreatment experience, $F(2,322)=4.717, M S E=0.322, p=.010$. Post hoc follow-up LSD tests demonstrated that the nonmaltreated comparison children performed significantly better than both those who were maltreated in their current developmental period $(M=0.44, S D=0.86, p=.004)$ and those who experienced maltreatment in the past $(M=0.23, S D=$ $0.71, p=.05)$. The performance of children who experienced maltreatment in their current developmental period compared to those who experienced it in previous periods was not statistically different from each other.

\section{Discussion}

The findings of this investigation reveal that maltreated children have a different cognitive control landscape than their nonmaltreated peers. On measures of inhibitory control and working memory, two constructs important for self-control (Moffitt et al., 2011; Weintraub et al., 2013; Zelazo et al., 2013), maltreated children exhibited poorer performance compared to nonmaltreated children. Finding this effect of maltreatment on children's accuracy and reaction time for tasks dependent on self-control mechanisms merits further consideration of the ways maltreatment negatively influences a child's later development. An increased focus on specific characteristics of maltreatment could help researchers further examine the effects of maltreatment at sensitive developmental periods. This nuanced approach to maltreatment research, consistent with the developmental psychopathology principle that the study of normal and abnormal development are mutually influential (Cicchetti \& Toth, 2009, in press), also will inform the broader field of self-control research by providing additional support for the importance of considering the early impact of adversity on developing self-control mechanisms.

A consideration of how both onset and duration of maltreatment significantly impact cognitive control performance (both inhibitory control and working memory) provides a rich account of how early experiences may inform the development of self-control processes. For example, discovering that children maltreated during infancy had significantly worse performance than children maltreated later in childhood indicates that timing of adversity matters in the development of self-control. The rapid neuronal changes during the first year of life may provide one explanation for this difference, because this period of neuronal growth is thought to increase the brain's sensitivity to external perturbations (Johnson, 2011).

Children may be especially vulnerable to the effects of maltreatment or other pathological experiences during periods of rapid creation or modification of neuronal connections such as infancy. Abnormal experiences may thus become part of a vicious cycle, whereby the pathology induced in brain structure may distort the child's experience and lead to subse- quent alterations in cognition or social interaction, thereby causing additional pathological experience and additional brain abnormality (Black, Jones, Nelson, \& Greenough, 1998; Cicchetti \& Tucker, 1994). Early maltreatment may condition young neural networks to produce cascading effects through later development, possibly constraining the child's flexibility to adapt to novel challenging situations with new strategies rather than with old conceptual and behavioral prototypes (Cicchetti \& Tucker, 1994; Courchesne, Chisum, \& Townsend, 1994; Masten \& Cicchetti, 2010). Maltreatment in infancy represents a clear case of early life stress, because it exceeds the infant's coping resources and increases the amount of stress experienced at a sensitive period of development (Pechtel \& Pizzagalli, 2011). This early stress increases the density of glucocorticoid receptors (Teicher et al., 2003). The increase in glucocorticoid receptors is theorized to affect the early maturation of the PFC, compromising the development of associated capacities (Teicher et al., 2003), as was evident in the diminished performance of children maltreated during infancy.

Early onset of maltreatment not only significantly predicted inhibitory control and working memory performance but also the chronicity of maltreatment. Children who experienced maltreatment during a single period of development performed as well as nonmaltreated children, whereas children who experienced chronic maltreatment (during $3+$ periods of development) performed significantly worse. This finding speaks to the importance of articulating the impact of maltreatment during the early years of life and highlights the deleterious effects of prolonged exposure to maltreatment. For example, if maltreatment occurred solely during one period of development, then the child's inhibitory control and working-memory capacities remained intact, more closely resembling a nonmaltreated child's profile than that of a child who experienced chronic maltreatment. Although nonmaltreated children performed significantly better than children who were maltreated during three or more periods of development, nonmaltreated children's performance was not better than children who only experienced maltreatment during one period of development. A longer history of maltreatment results in prolonged period of stress on the child's neuroendocrine and immune systems and possible neural reorganization to optimize survival in a deprived and difficult environment (Johnson, 2011; Teicher et al., 2003). Numerous interconnected neurobiological systems are affected by the various stressors associated with child maltreatment (DeBellis, 2001, 2005; Hart \& Rubia, 2012). Moreover, each of these neurobiological systems influences and is influenced by multiple domains of biological and psychological development. A possible explanation for our results may have to do with the understanding that inhibitory control and workingmemory tasks rely on the recruitment of the PFC (Diamond, 1997). The density of the synapses in the PFC increases slowly over the course of development, and the experience of prolonged maltreatment may compromise synaptogenesis (Johnson, 2011). 
The child-caregiver relationship may also play an important role because the precursor of higher cognition during infancy is connecting an act to a consequence. A phenomena such as joint attention, in which an infant and caregiver both attend to the same stimuli (Tomasello \& Akhtar, 1995), presents an infant with the experience that cues, such as eye gaze, are important for knowing what to attend to in his or her environment. For maltreated children, however, a relatively straightforward act of connecting a cue to a consequence may be difficult, given unpredictability and inconsistency in the maltreating family environment. Major occurrences in the life of maltreated children, such as abuse or the withholding of care, result in a pathogenic, chaotic, and threatening environment that negatively influences their ability to develop basic cognitive skills.

If children were chronically maltreated, then their performance on the inhibitory control and working-memory tasks decreased significantly. Examining the chronicity and onset of maltreatment provided insight into a child's inhibitory control and working-memory development. An examination of the number of maltreatment subtypes a child experienced did not yield incremental information beyond the basic statement that children who had experienced one to four maltreatment subtypes (i.e., all maltreated children) performed worse than nonmaltreated children. A possible explanation is that only a minority of maltreated children experienced three or more maltreatment subtypes. In our sample, 153 children experienced one or two maltreatment subtypes, and only 34 children experienced three or more maltreatment subtypes.

The findings of the current research illustrate diversity among maltreated children in their acquisition of executive function skills. Maltreatment during the infancy period and chronic maltreatment were found to have importance for identifying which maltreated children were most vulnerable to impairments in the development of inhibitory control and working-memory abilities. These results suggest that infancy is a sensitive period that is critical for establishing the foundations of brain structure and organization involved in the development of later executive function abilities. Moreover, chronic maltreatment further impairs executive function development. These insights also are important for understanding the early precursors necessary for executive function development in normative rearing conditions.

A limitation of the current investigation is the cross-sectional nature of the design with assessment of children at only one point in time. Future directions include longitudinally examining the relationship between maltreatment and inhibitory control and working memory. It would be useful to

\section{References}

Balamore, U., \& Wozniak, R. H. (1984). Speech-action coordination in young children. Developmental Psychology, 20, 850-858.

Barnett, D., Manly, J. T., \& Cicchetti, D. (1993). Defining child maltreatment: The interface between policy and research. In D. Cicchetti \& S. L. Toth (Eds.), Child abuse, child development, and social policy ( $\mathrm{pp}$. 7-74). Norwood, NJ: Ablex understand when these discrepancies emerge and whether performance decrements increase as a function of maltreatment and lessen when a child transitions to adulthood. If maltreatment decreases over the course of childhood and ends by adulthood, then it would be informative to see if the growing child is able to incorporate new experiences and develop a clear sense of agency. Alternatively, the early foundation of disrupted inhibitory control and working memory may lead to further disruptions in higher order processes that affect related processes, such as the formation of relationships and the ability to maintain employment. A first step in extending this work would be to examine the relationship between maltreatment and executive function in adolescence and to consider executive function in either a cool or hot context (Zelazo \& Carlson, 2012). One way to accomplish this would be to conduct functional magnetic resonance imaging research with maltreated youth using inhibitory control and working-memory tasks.

Overall, it is clear that the onset and chronicity of maltreatment plays a significant role in the early stages of workingmemory and inhibitory control development. This likely influences how well a child performs in the classroom. At the same time, the cognitive abilities of children who experienced maltreatment later during development or who experienced maltreatment for a lesser portion of development may be indistinguishable from their SES-matched nonmaltreated counterparts. For these reasons, it is important to consider the role of multifinality in childhood maltreatment and to acknowledge that children will experience and express the consequences of maltreatment differently, which is in part a reflection of their maltreatment experience (Cicchetti, 2010, 2013). Future intervention research concerned with promoting the development of self-control in maltreated children may benefit from focusing efforts on groups of children who are particularly vulnerable to perturbations in self-control, such as those experiencing significant adversity during infancy or those chronically exposed to harmful environments. Given the findings on the deleterious effects of maltreatment during infancy (Cicchetti \& Lynch, 1993; Cicchetti \& Valentino, 2006), it will be particularly important to focus on how best to provide intervention to these vulnerable infants in hopes of diverting them from a negative to a more positive developmental trajectory. In addition, once maltreatment has occurred, intervention efforts should be directed toward ensuring that a pattern of chronic maltreatment does not occur. Toward this end, ideally child welfare systems should develop a mechanism for supporting positive parenting even after an active CPS case has been closed.
Berman, M. G., Yourganov, G., Askren, M. K., Ayduk, O., Casey, B. J., Gotlib, I. H., et al. (2013). Dimensionality of brain networks linked to life-long individual differences in self-control. Nature Communications, 4, 1-7.

Black, J., Jones, T. A., Nelson, C. A., \& Greenough, W. T. (1998). Neuronal plasticity and the developing brain. In N. E. Alessi, J. T. Coyle, S. I. Har- 
rison, \& S. Eth (Eds). Handbook of child and adolescent psychiatry (pp. 31-53). New York: Wiley.

Blair, C., Raver, C. C., \& Berry, D. J. (2014). Two approaches to estimating the effect of parenting on the development of executive function in early childhood. Developmental Psychology, 50, 554-565.

Bolger, K. E., \& Patterson, C. J. (2001). Developmental pathways from child maltreatment to peer rejection. Child Development, 72, 549-568.

Bruce, J., Fisher, P. A., Graham, A. M., Moore, W. E., Peake, S. J., \& Mannering, A. M. (2013). Patterns of brain activation in foster children and nonmaltreated children during an inhibitory control task. Development and Psychopathology, 25, 931-941.

Casey, B. J., Somerville, L. H., Gotlib, I. H., Ayduk, O., Franklin, N. T., Askren, M. K., et al. (2011). Behavioral and neural correlates of delay of gratification 40 years later. Proceedings of the National Academy of Sciences, 108, 14998-5003.

Chugani, H. T., Behen, M. E., Muzik, O., Juhâsz, C., Nagy, F., \& Chugani, D. C. (2001). Local brain functional activity following early deprivation: A study of postinstitutionalized Romanian orphans. NeuroImage, 14, $1290-1301$.

Cicchetti, D. (2010). Resilience under conditions of extreme stress: A multilevel perspective. World Psychiatry, 9, 145-154.

Cicchetti, D. (2013). Annual Research Review: Resilient functioning in maltreated children-Past, present, and future perspectives. Journal of Child Psychology and Psychiatry, 54, 402-422.

Cicchetti, D., \& Barnett, D. (1991). Attachment organization in maltreated preschoolers. Development and Psychopathology, 3, 397-411.

Cicchetti, D., \& Lynch, M. (1993). Toward an ecological/transactional model of community violence and child maltreatment: Consequences for children's development. Psychiatry, 56, 96-118.

Cicchetti, D., \& Manly, J. T. (Eds.) (2001). Operationalizing child maltreatment: Developmental processes and outcomes [Special Issue]. Development and Psychopathology, 13, 755-1048.

Cicchetti, D., \& Rogosch, F. A. (1996). Equifinality and multifinality in developmental psychopathology. Development and Psychopathology, 8, 597-600.

Cicchetti, D., \& Toth, S. L. (1995). A developmental psychopathology perspective on child abuse and neglect. Journal of the American Academy of Child \& Adolescent Psychiatry, 34, 541-565.

Cicchetti, D., \& Toth, S. L. (2009). The past achievements and future promises of developmental psychopathology: The coming of age of a discipline. Journal of Child Psychology and Psychiatry, 50, 16-25.

Cicchetti, D., \& Toth, S. L. (in press). Child maltreatment. In M. Lamb \& C. Garcia Coll (Eds.), Handbook of child psychology and developmental science: Vol. 3. Socioemotional process (7th ed.). Hoboken, NJ: Wiley.

Cicchetti, D., Toth, S. L., \& Manly, J. T. (2003). Maternal Maltreatment Classification Interview. Unpublished manuscript, Mt Hope Family Center, Rochester, NY.

Cicchetti, D., \& Tucker, D. (1994). Development and self-regulatory structures of the mind. Development and Psychopathology, 6, 533-549.

Cicchetti, D., \& Valentino, K. (2006). An ecological transactional perspective on child maltreatment: Failure of the average expectable environment and its influence upon child development. In D. Cicchetti \& D. J. Cohen (Eds.), Developmental psychopathology (Vol. 3, 2nd ed., pp. 129-201). Hoboken, NJ: Wiley.

Courchesne, E., Chisum, H., \& Townsend, J. (1994). Neural activity-dependent brain changes in development: Implications for psychopathology. Development and Psychopathology, 6, 697-722.

DeBellis, M. D. (2001). Developmental traumatology: The psychobiological development of maltreated children and its implications for research, treatment, and policy. Development and Psychopathology, 13, 539-564.

De Bellis, M. D. (2005). The psychobiology of neglect. Child Maltreatment, 10, 150-172.

De Bellis, M. D., Keshavan, M. S., Shifflett, H., Iyengar, S., Beers, S. R., Hall, J., et al. (2002). Brain structures in pediatric maltreatment-related posttraumatic stress disorder: A socio- demographically matched study. Biological Psychiatry, 52, 1066-1078.

Diamond, A., Prevor, M. B., Callender, G., \& Druin, D. P. (1997). Prefrontal cortex cognitive deficits in children treated early and continuously for PKU. Monographs of the Society for Research in Child Development, 62, 1-206.

Diamond, A., \& Taylor, C. (1996). Development of an aspect of executive control: Development of the abilities to remember what I said and to "Do as I say, not as I do." Developmental Psychobiology, 29, 315-334.
Eigsti, I. M., Zayas, V., Mischel, W., Shoda, Y., Ayduk, O., Dadlani, M. B., et al. (2006). Predicting cognitive control from preschool to late adolescence and young adulthood. Psychological Science, 17, 478-484.

Fox, S. E., Levitt, P., \& Nelson, III, C. A. (2010). How the timing and quality of early experiences influence the development of brain architecture. Child Development, 81, 28-40.

Gerstadt, C. L., Hong, Y. J., \& Diamond, A. (1994). The relationship between cognition and action: Performance of children $31 / 2-7$ years old on a Stroop-like day-night test. Cognition, 53, 129-153.

Hanson, J. L., Adluru, N., Chung, M. K., Alexander, A. L., Davidson, R. J., \& Pollak, S. D. (2013). Early neglect is associated with alterations in white matter integrity and cognitive functioning. Child Development, $84,1566-1578$.

Hare, T. A., Tottenham, N., Galvan, A., Voss, H. U., Glover, G. H., \& Casey, B. J. (2008). Biological substrates of emotional reactivity and regulation in adolescence during an emotional go-no-go task. Biological Psychiatry, 63, 927-934.

Hart, H., \& Rubia, K. (2012). Neuroimaging of child abuse: A critical review. Frontiers in Human Neuroscience, 6, 1-24.

Johnson, M. H. (2011). Interactive specialization: A domain-general framework for human functional brain development? Developmental Cognitive Neuroscience, 1, 7-21.

Lupien, S. J., McEwen, B. S., Gunnar, M. R., \& Heim, C. (2009). Effects of stress throughout the lifespan on the brain, behaviour and cognition. $\mathrm{Na}$ ture Reviews Neuroscience, 10, 434-445.

Manly, J. T., Kim, J. E., Rogosch, F. A., \& Cicchetti, D. (2001). Dimensions of child maltreatment and children's adjustment: Contributions of developmental timing and subtype. Development and Psychopathology, 13, 759-782.

Marshall, P. J., \& Fox, N. A. (2004). A comparison of the electroencephalogram between institutionalized and community children in Romania. Journal of Cognitive Neuroscience, 16, 1327-1338.

Masten, A. S., \& Cicchetti, D. (2010). Developmental cascades. Development and Psychopathology, 22, 491-495.

Mathew, S. J., Coplan, J. D., Smith, E. L., Scharf, B. A., Owens, M. J., Nemeroff, C. B., et al. (2002). Cerebrospinal fluid concentrations of biogenic amines and corticotropin-releasing factor in adolescent non-human primates as a function of the timing of adverse early rearing. Stress, 5, 185-193.

McCrory, E., De Brito, S. A., \& Viding, E. (2010). Research review: The neurobiology and genetics of maltreatment and adversity. Journal of Child Psychology and Psychiatry, 51, 1079-1095.

McCrory, E., \& Viding, E. (2010). The neurobiology of maltreatment and adolescent violence. Lancet, 375, 1856-1857.

McDermott, J. M., Westerlund, A., Zeanah, C. H., Nelson, C. A, \& Fox, N. A. (2012). Early adversity and neural correlates of executive function: Implications for academic adjustment. Developmental Cognitive Neuroscience, 2, S59-S66.

Mischel, W., Ayduk, O., Berman, M. G., Casey, B. J., Gotlib, I. H., Jonides, J., et al. (2010). "Willpower" over the life span: Decomposing self-regulation. Social Cognitive and Affective Neuroscience, 6, 252-256.

Moffitt, T. E., Arseneault, L., Belsky, D., Dickson, N., Hancox, R. J., Harrington, H., et al. (2011). A gradient of childhood self-control predicts health, wealth, and public safety. Proceedings of the National Academy of Sciences, 108, 2693-2698.

Pechtel, P., \& Pizzagalli, D. A. (2011). Effects of early life stress on cognitive and affective function: An integrated review of human literature. Psychopharmacology, 214, 55-70.

Sedlak, A. J., Mettenburg, J., Basena, M., Petta, I., McPherson, K., Greene, A., et al. (2010). Fourth National Incidence Study of Child Abuse and Neglect (NIS-4): Report to Congress, executive summary. Washington, DC: US Department of Health and Human Services, Administration for Children and Families.

Sroufe, L. (1990). Considering normal and abnormal together: The essence of developmental psychopathology. Development and Psychopathology, 2, 335-347.

Sroufe, L. A., Egeland, B., Carlson, E. A., \& Collins, W. A. (2005). The development of the person: The Minnesota study of risk and adaptation from birth to adulthood. New York: Guilford Press.

Stiles, J., \& Jernigan, T. L. (2010). The basics of brain development. Neuropsychology Review, 20, 327-348.

Sullivan, R. M. (2003). Developing a sense of safety. Annals of the New York Academy of Sciences, 1008, 122-131.

Teicher, M. H., Anderson, C. M., \& Polcari, A. (2012). Childhood maltreatment is associated with reduced volume in the hippocampal subfields 
CA3, dentate gyrus, and subiculum. Proceedings of the National Academy of Sciences, 109, E563-E572.

Teicher, M. H., Andersen, S. L., Polcari, A., Anderson, C. M., Navalta, C. P., \& Kim, D. M. (2003). The neurobiological consequences of early stress and childhood maltreatment. Neuroscience \& Biobehavioral Reviews, 27, 33-44.

Thompson, R. A., \& Nelson, C. A. (2001). Developmental science and the media: Early brain development. American Psychologist, 56, 5-15.

Thornberry, T. P., Ireland, T. O., \& Smith, C. A. (2001). The importance of timing: The varying impact of childhood and adolescent maltreatment on multiple problem outcomes. Development and Psychopathology, 13, 957-979.

Tomasello, M., \& Akhtar, N. (1995). Two-year-olds use pragmatic cues to differentiate reference to objects and actions. Cognitive Development, 10, 201-224.

Weintraub, S., Dikmen, S. S., Heaton, R. K., Tulsky, D. S., Zelazo, P. D., Bauer, P. J., et al. (2013). Cognition assessment using the NIH Toolbox. Neurology, 80, S54-S64.
Zelazo, P. D., Anderson, J. E., Richler, J., Wallner-Allen, K., Beaumont, J. L., \& Weintraub, S. (2013). In NIH toolbox cognition battery (CB): Measuring executive function and attention. Monographs of the Society for Research in Child Development, 78, 16-33.

Zelazo, P. D., \& Bauer, P. J. (Eds). (2013). National Institutes of Health Toolbox-Cognition Battery (NIH Toolbox CB): Validation for children between 3 and 15 years. Monographs of the Society for Research in Child Development, 78, 16-33.

Zelazo, P. D., \& Carlson, S. M. (2012). Hot and cool executive function in childhood and adolescence: Development and plasticity. Child Development Perspectives, 6, 354-360.

Zelazo, P. D., Carlson, S. M., \& Kesel, A. (2008). The development of executive function in childhood. In C. Nelson \& M. Luciana (Eds.), Handbook of developmental cognitive neuroscience (2nd ed., pp. 533-574). Cambridge, MA: MIT Press. 